\title{
Influência do monitoramento de indicadores e informações de saúde bucal no desempenho das equipes de saúde bucal em estado Amazônico
}

\author{
Influence of oral health indicators and information monitoring on the performance of oral \\ health teams in an Amazon state
}

Influencia del monitoreo de indicadores y información sobre salud bucal en el desempeño de los equipos de salud bucal en el estado Amazónico

Gabriel Mácola de Almeida ${ }^{1}$, Pettra Blanco Lira Matos ${ }^{1}$, Lucas Oliveira da Silva ${ }^{1}$, Diandra Costa Arantes ${ }^{1}$, Adalberto Lírio de Nazaré Lopes ${ }^{1}$, Rosana Nazaré Leão Souza ${ }^{1}$, Cecília Abrahão Nascimento de Santi ${ }^{1}$, Flávia Sirotheau Corrêa Pontes ${ }^{1}$, Helder Antônio Rebelo Pontes ${ }^{1}$, Liliane Silva do Nascimento ${ }^{1 *}$.

\section{RESUMO}

Objetivo: Verificar a situação e impacto do monitoramento e avaliação dos indicadores e informações de saúde bucal sobre o desempenho das Equipes de Saúde Bucal (ESB) no estado do Pará. Métodos: Estudo ecológico descritivo que utilizou dados secundários das ESB participantes do $2^{\circ}$ ciclo do PMAQ-AB Pará. Para avaliação da relação entre o monitoramento de indicadores e o desempenho das ESB, foram utilizados os testes qui-quadrado e regressão logística multinominal. Resultados: Participaram 500 ESB. A maioria das equipes não realiza monitoramento e análise dos indicadores e informações de saúde bucal $(245 ; 49,0 \%)$, tendendo a apresentar desempenho regular e insuficiente. Cento e oitenta e três $(36,6 \%)$ equipes recebem apoio para a discussão dos dados de monitoramento, apresentando diferenças estatisticamente significativas entre as equipes que recebem e as que não recebem este apoio $(p<0,05)$. Verificou-se que as ESB que realizam monitoramento dos indicadores têm 8,86 mais chances de receber um bom desempenho e 29,85 mais chances de apresentar um ótimo desempenho. Conclusão: Houve impacto positivo no desempenho das ESB que realizaram e receberam apoio no monitoramento dos indicadores, reafirmando esta prática como ferramenta fortalecedora e necessária dos serviços de atenção à saúde.

Palavras chave: Indicadores básicos de saúde, Avaliação de desempenho profissional, Saúde bucal, Atenção primária à saúde.

\section{ABSTRACT}

Objective: To verify the situation and impact of monitoring and evaluation of indicators and information of oral health on the performance of Oral Health Teams (OHT) in the state of Pará. Methods: Descriptive ecological study that used secondary data from OHT participants of the 2nd cycle of PMAB-AB Pará, collected on the period from March to April 2019. To evaluate the association between indicators monitoring and the $\mathrm{OHT}$ performance, chi-square and logistic regression analysis were carried out. Results: $500 \mathrm{OHT}$ participated. Great majority of the teams does not monitor and analyze oral health indicators and information $(245 ; 49,0 \%)$, tending to present regular and insufficient performance. $183(36,6 \%)$ teams receive support to discuss monitoring data, presenting statistically significant differences between the teams that receive and do not receive this support $(p<0.05)$. It was found that the $\mathrm{OHT}$ that perform the monitoring of the indicators are 8.86 more likely to receive a good performance and 29.85 more likely to present an excellent performance. Conclusion: There is a positive impact on the OHT that carry out and are supported to monitor indicators, reaffirming this exercise as a strengthening tool and necessary in the health care services.

Key words: Health status indicators, Employee performance appraisal, Oral health, Primary health care.

1Universidade Federal do Pará (UFPA), Belém - PA. *E-mail: profaliliane@ufpa.br 


\section{RESUMEN}

Objetivo: Verificar la situación y el impacto del monitoreo y la evaluación de los indicadores e información de salud bucal sobre el desempeño de los Equipos de Salud Oral (ESB) en el estado de Pará. Métodos: Estudio ecológico descriptivo que utilizó datos secundarios de los ESB de segundo ciclo PMAB-AB Pará. Para evaluar la relación entre monitoreo de indicadores y el desempeño de los ESB, se utilizaron las pruebas de chicuadrado y regresión logística multinominal. Resultados: Participaron 500 ESB. La mayoría de los equipos no realizan monitoreo y análisis de indicadores e información de salud bucal $(245 ; 49,0 \%)$, tendiendo a tener un desempeño regular y insuficiente. $183(36,6 \%)$ equipos reciben apoyo para la discusión de los datos de monitoreo, mostrando diferencias estadísticamente significativas entre los equipos que reciben y no reciben apoyo $(\mathrm{p}<0.05)$. Los ESB que realizan monitoreo de indicadores tienen 8.86 más probabilidades de recibir un buen desempeño y 29.85 más probabilidades de tener un excelente desempeño. Conclusión: Hay un impacto positivo en el desempeño de los ESB que realizan y reciben apoyo en el monitoreo de los indicadores, reafirmando esta práctica como una herramienta de fortalecimiento y necesaria de los servicios de salud.

Palabras clave: Indicadores de salud, Evaluación del rendimiento de empleados, Salud bucal, Atención primaria de salud.

\section{INTRODUÇÃO}

A instituição da Política Nacional de Saúde Bucal (PNSB) representa grande avanço para a Odontologia no Brasil, ampliando o acesso à assistência odontológica para a população brasileira. Esta, em conjunto com a Política Nacional de Atenção Básica (PNAB), estabelece diretrizes para a organização do processo de trabalho e do cotidiano das Equipes de Saúde Bucal (ESB) (BRASIL, 2004; BRASIL, 2017).

Segundo a PNSB, o cirurgião-dentista deve atuar na promoção de saúde, prevenção de doenças, tratamento e reabilitação oral, realizando atendimentos, planejamento e participação em ações coletivas direcionadas à população e atuação conjunta com os profissionais de outras equipes. Além disto, profissional de saúde bucal deve acompanhar o impacto dessas atividades por meio de indicadores adequados, incorporando práticas contínuas de monitoramento e avaliação de danos, riscos e determinantes do processo saúde-doença (BRASIL, 2004).

Os indicadores de saúde são uma forma de medida-síntese que possibilitam visualizar as características da realidade do estado de saúde de uma determinada população e do próprio sistema de saúde vigente, sendo facilitadores para a manutenção e o planejamento em saúde. Por conter informações relevantes sobre esses cenários, os indicadores contribuem para a criação de novas políticas públicas e 0 ajuste de prioridades que atentem para as necessidades da população (RIPSA, 2008; FRANÇA MASA, et al., 2018).

Através da prática do monitoramento de indicadores, é possível avaliar as atividades e processos realizados pela equipe, bem como planejar adequadamente ações futuras, melhorando gradativamente a qualidade da atenção à saúde para a população. Geram-se subsídios para o planejamento de intervenções e a criação de estratégias que superem as dificuldades e permitam o crescimento contínuo da Atenção Primária ao passo que colaboram para a consolidação da Estratégia da Saúde da Família (LIMA CA, et al., 2019).

Estes indicadores também são essenciais para o acompanhamento da produção do cuidado exercida pelas equipes e a forma como estas se organizam em seu processo de trabalho. Ao longo dos anos, o uso de indicadores tornou possível analisar a quantidade e a qualidade dos serviços de saúde bucal prestados e determinar se eles são adequados e suficientes para atender à demanda existente.

Os indicadores mostram se as ações planejadas devem ser aprimoradas e alteradas e determinam o nível de conformidade dessas ações com os princípios do Sistema Único de Saúde (SUS), entre outras informações (GONÇALVES J e SAMPAIO J, 2015; FRANÇA MASA, et al., 2018).

O monitoramento é uma ferramenta estratégica de gestão para acompanhar informações prioritárias que refletem no processo de resolutividade, ou a falta desta, das intervenções adotadas, funcionando também como norteador na tomada de decisões. Nesse sentido, os sistemas de informação são a plataforma de acesso para estas informações. Em saúde bucal, os sistemas nacionais usados são o Sistema de Informação 
da Atenção Básica (SIAB) e o Sistema de Informações Ambulatoriais do SUS (SIA-SUS). A análise dos indicadores coletados destes bancos de dados tem relevância significativa para a avaliação dos impactos das ações em saúde em âmbito municipal, estadual e federal (GONÇALVES J e SAMPAIO J, 2015; ZERMIANI TC, et al., 2014).

O contínuo monitoramento de indicadores é imperativo na medida em que permite o direcionamento de atenção e recursos para áreas com maiores necessidades, auxiliando na implementação de serviços de saúde apropriados para aquela região. Idealiza-se, assim, a construção de um padrão de processo de trabalho que atenda às necessidades de saúde na oferta de serviços de qualidade atuando em diferentes cenários, gerando avanços na efetividade do cuidado e evidenciando as potencialidades, fragilidades para a superação de desafios (ZERMIANI TC, et al., 2014; NEVES M, et al., 2019).

Nesse horizonte, reafirma-se a necessidade de avaliar a importância dos impactos tanto em saúde bucal quanto nas próprias equipes de profissionais de saúde no processo de monitoramento desses indicadores de saúde.

Dessa forma, o presente estudo teve como objetivo verificar a situação e o impacto do monitoramento e da avaliação dos indicadores e das informações de saúde bucal sobre o desempenho das equipes de saúde bucal no estado do Pará.

\section{MÉTODOS}

Este estudo ecológico descritivo teve como base os dados secundários das ESB participantes do $2^{\circ}$ ciclo do Programa Nacional de Melhoria do Acesso e da Qualidade da Atenção Básica (PMAQ-AB) no estado do Pará. O estudo segue os princípios da ética em pesquisa definidos pela resolução 466/2012, e por utilizar de dados públicos disponíveis em bases de dados nacionais, dispensa o uso de Termo de Consentimento Livre e Esclarecido.

O PMAQ-AB é um programa nacional de cunho avaliativo que acontece em ciclos e cuja finalidade é estimular gestores e equipes de saúde a aprimorar a qualidade da atenção ofertada à população. Por meio do método de pagamento por desempenho, o programa proporciona repasse de recursos como forma de incentivo para os municípios contratualizados, que varia conforme o desempenho (certificação) alcançado dentro das metas do próprio instrumento de avaliação (MINISTÉRIO DA SAÚDE, 2013).

$O$ PMAQ-AB é desenvolvido em fases. No começo do ciclo, a primeira fase é a adesão, em que os compromissos vinculados ao programa são pactuados entre as equipes, os gestores municipais e o Ministério da Saúde (MS). A segunda fase consiste na adoção de ações de desenvolvimento, cujo intuito é de gerar mudanças positivas na gestão do cuidado através das dimensões de autoavaliação, monitoramento, educação permanente e apoio institucional (MINISTÉRIO DA SAÚDE, 2013; SEIDL H, et al., 2014; FLÔRES GMS, et al., 2018).

Durante a terceira fase, chamada de avaliação externa, com o objetivo de verificar os padrões de qualidade, de infraestrutura e de acesso, os usuários e os profissionais de saúde são entrevistados por equipes técnicas do programa, capacitadas para aplicar o instrumento de avaliação em campo. O instrumento de entrevista utilizado na avaliação externa foi padronizado em aplicativo (software) para tablets.

Todos os entrevistadores foram previamente preparados através de treinamento para realizar as visitas de campo e as entrevistas nas unidades de saúde. No caso das unidades de saúde com ESB, um dos cirurgiões-dentistas cadastrados à unidade e presente no momento da avaliação responde às perguntas $e$ representa a equipe (MINISTÉRIO DA SAÚDE, 2013; SEIDL H, et al., 2014; FLÔRES GMS, et al., 2018).

$\mathrm{Na}$ quarta e última fase, ocorre o processo de certificação de desempenho das equipes participantes, levando em consideração os dados coletados. Após a avaliação e conforme o desempenho, as equipes podem obter certificação "Ótimo", "Bom", "Regular", "Insuficiente" e "Desclassificado". As equipes participantes do PMAQ-AB cujo cirurgião-dentista não estava presente para responder a entrevista do programa na fase de avaliação externa ou se recusou a realizar a mesma automaticamente entraram na categoria de certificação "Desclassificado" pelo próprio programa (MINISTÉRIO DA SAÚDE, 2013; SEIDL H, et al., 2014; FLÔRES GMS, et al., 2018). 
Neste estudo, foram utilizados os dados obtidos a partir das entrevistas com as ESB na terceira fase do $2^{\circ}$ ciclo do PMAQ-AB, a de avaliação externa, além dos dados referentes às certificações geradas após o encerramento do ciclo. O 2ํㅜㅇㅣ ciclo do PMAQ-AB ocorreu em 2014 e seus dados, disponibilizados nos anos de 2016 e 2017, constituem arquivo disponível no portal eletrônico do MS.

A coleta de dados para esta pesquisa ocorreu no período de março a abril de 2019 através do portal da Secretaria de Atenção Primária à Saúde (SAPS), onde foram disponibilizados como domínio público pelo MS. Os conceitos de desempenho por unidade nos municípios do estado do Pará, resultados da fase final de certificação do programa, foram coletados no portal do e-gestor (E-Gestor, 2017).

Foram avaliadas 30.522 equipes de atenção básica em todo território nacional. No estado do Pará, 794 equipes de saúde foram contratualizadas, sendo 500 ESB. Constituíram a amostra desta pesquisa todas as 500 ESB. Foram excluídas do estudo as ESB não contratualizadas no ciclo do PMAQ-AB em questão.

Além da variável certificação, foram analisadas na pesquisa duas variáveis coletadas do módulo VI do instrumento de avaliação externa. O módulo VI corresponde à entrevista com profissional de saúde bucal da ESB, com perguntas voltadas ao processo de trabalho da equipe, ao perfil dos profissionais e à documentação da unidade.

As perguntas desse módulo incluídas como variáveis foram: 1- "A Equipe de Saúde Bucal realiza monitoramento e análise dos indicadores e informações de saúde bucal?" e 2- "A equipe recebe apoio para a discussão dos dados de monitoramento do sistema de informação?".

A fim de verificar se houve diferença de certificação obtida entre as ESB que realizaram ou não monitoramento e análise dos indicadores de saúde bucal e que receberam ou não apoio para discussão dos dados do monitoramento, foi utilizado o teste qui-quadrado.

Para avaliar se houve associação entre a realização de monitoramento e análise de indicadores e a certificação obtida pelas ESB, foi aplicado o teste de regressão logística multinomial. Para ambas as análises, foi utilizado o software SPSS Statistics (Versão 23.0. Armonk, NY: IBM Corp).

\section{RESULTADOS}

Os dados obtidos mostraram que a maioria das ESB recebeu certificação "Regular" nos resultados da fase final do $2^{\circ}$ ciclo do PMAQ-AB (Tabela 1).

Tabela 1 - Distribuição do desempenho dos profissionais da Equipe de Saúde Bucal por certificação recebida, no estado do Pará. 2019.

\begin{tabular}{lcc}
\hline Certificação & Frequência & Percentual (\%) \\
\hline Ótimo & 12 & 2,4 \\
Bom & 64 & 12,8 \\
Regular & 342 & 68,4 \\
Insuficiente & 26 & 5,2 \\
Desclassificado & 56 & 11,2 \\
\hline Total & $\mathbf{5 0 0}$ & $\mathbf{1 0 0 , 0}$ \\
\hline
\end{tabular}

Fonte: Almeida GM, et al., 2020. Microdados da avaliação externa do PMAQ-AB (2014).

A Tabela 2 evidencia que, no estado do Pará, a maioria das ESB não realizou monitoramento e análise dos indicadores e informações de saúde bucal. Houve diferença estatisticamente significativa entre as ESB que realizaram e que não realizaram monitoramento e análise dos indicadores e das informações em saúde bucal $(p<0,001)$. As ESB que não realizaram monitoramento e análise dos indicadores e informações de saúde bucal tendem a apresentar certificação "Regular" (205; 59,9\%) e "Insuficiente" (19; 73,1\%).

A análise de regressão logística multinominal sugeriu que equipes que realizaram monitoramento e análise dos indicadores e informações de saúde bucal, em comparação às que não realizaram, apresentaram aproximadamente vinte e nove vezes mais chance de obter certificação "Ótimo" e oito vezes mais chance de obter certificação "Bom" do que a certificação "Insuficiente" (Tabela 3). 
Tabela 2 - Distribuição dos profissionais da Equipe de Saúde Bucal, segundo o desempenho em função do monitoramento e análise dos indicadores e informações de saúde bucal da Equipe de Saúde Bucal, no estado do Pará. 2019.

\begin{tabular}{|c|c|c|c|c|c|c|c|c|c|c|c|c|}
\hline \multirow{3}{*}{$\begin{array}{l}\text { A Equipe de Saúde } \\
\text { Bucal realiza } \\
\text { monitoramento e } \\
\text { análise dos } \\
\text { indicadores e } \\
\text { informações de saúde } \\
\text { bucal? }\end{array}$} & \multicolumn{10}{|c|}{ Certificação } & \multirow{3}{*}{$\mathrm{X}^{2}$} & \multirow{3}{*}{$p$ valor } \\
\hline & \multicolumn{2}{|c|}{$\begin{array}{c}\text { Ótimo } \\
(n=12)\end{array}$} & \multicolumn{2}{|c|}{$\begin{array}{c}\text { Bom } \\
(n=64)\end{array}$} & \multicolumn{2}{|c|}{$\begin{array}{c}\text { Regular } \\
(\mathrm{n}=342)\end{array}$} & \multicolumn{2}{|c|}{$\begin{array}{c}\text { Insuficient } \\
\mathrm{e} \\
(\mathrm{n}=26) \\
\end{array}$} & \multicolumn{2}{|c|}{$\begin{array}{c}\text { Desclassificad } \\
0 \\
(n=56) \\
\end{array}$} & & \\
\hline & $\mathrm{n}$ & $\%$ & $\mathrm{n}$ & $\%$ & $\mathrm{n}$ & $\%$ & $\mathrm{n}$ & $\%$ & $\mathrm{n}$ & $\%$ & & \\
\hline Não se aplica & - & - & - & & - & - & - & - & 47 & 83,9 & & \\
\hline Sim & 11 & 91,7 & 49 & 76,6 & 137 & 40,1 & 7 & 26,9 & 4 & 7,1 & $\begin{array}{c}458,57 \\
?\end{array}$ & $<0,001$ \\
\hline Não & 1 & 8,3 & 15 & 23,4 & 205 & 59,9 & 19 & 73,1 & 5 & 8,9 & & \\
\hline
\end{tabular}

Fonte: Almeida GM, et al., 2020. Microdados da avaliação externa do PMAQ-AB (2014).

Tabela 3 - Modelo de Regressão Logística Multinominal para avaliação do desempenho dos profissionais da Equipe de Saúde Bucal, no estado do Pará. 2019.

\begin{tabular}{lcccc}
\multicolumn{1}{c}{ Certificação } & $\boldsymbol{\beta}$ & $\mathbf{p}$ valor & Odds ratio & $\begin{array}{c}\text { Intervalo de Confiança } \\
\mathbf{9 5 \%}\end{array}$ \\
\hline Ótimo & 1 & & & \\
\hline Não & 3,396 & 0,003 & 29,857 & $3,233-275,725$ \\
Sim & 1 & & & $3,128-25,132$ \\
\hline Bom & 2,182 & $<0,001$ & 8,867 & \\
\hline Não & & & & $0,743-4,431$ \\
Sim & 1 & & 1,814 & \\
\hline Regular & 0,595 & 0,191 & & \\
\hline Não & & & & \\
\hline
\end{tabular}

Categoria de referência: Certificação INSUFICIENTE

Fonte: Almeida GM, et al., 2020. Microdados da avaliação externa do PMAQ-AB (2014).

A Tabela 4 apresenta a distribuição dos profissionais da ESB, segundo o apoio da gestão para a discussão dos dados de monitoramento do sistema de informação no estado do Pará. Verificou-se que menos da metade das equipes receberam apoio para a discussão dos dados de monitoramento do sistema de informação. Todas as equipes que obtiveram certificação "Ótimo" receberam apoio para a discussão dos dados de monitoramento do sistema de informação, assim como a maioria das equipes que obtiveram certificação "Bom". As equipes que não receberam o apoio apresentaram a tendência a obter certificação "Regular".

Tabela 4 - Desempenho dos Profissionais da Equipe de Saúde Bucal, segundo o apoio da gestão para as Equipes de Saúde Bucal, no estado do Pará. 2019.

\section{Certificação}

Apoio da gestão

\begin{tabular}{ccccccccc}
\hline $\begin{array}{c}\text { Ótimo } \\
(\mathrm{n}=12)\end{array}$ & $\begin{array}{c}\text { Bom } \\
(\mathrm{n}=64)\end{array}$ & $\begin{array}{c}\text { Regular } \\
(\mathrm{n}=342)\end{array}$ & $\begin{array}{c}\text { Insuficiente } \\
(\mathrm{n}=26)\end{array}$ & $\begin{array}{c}\text { Desclassificado } \\
(\mathrm{n}=56)\end{array}$
\end{tabular} $\mathbf{X}^{2} \begin{gathered}\mathbf{p} \\
\text { valor }\end{gathered}$

A equipe recebe apoio para a discussão dos dados de monitoramento do sistema de informação?

\begin{tabular}{|c|c|c|c|c|c|c|c|c|c|c|c|}
\hline Sem avaliação externa & - & - & - & - & - & - & - & - & 47 & 83,9 & \\
\hline Sim & 12 & 100,0 & 43 & 67,2 & 114 & 33,3 & 7 & 26,9 & 7 & 12,5 & \\
\hline Não & - & - & 10 & 15,6 & 128 & 37,4 & 8 & 30,8 & 1 & 1,8 & $442,220<0,001$ \\
\hline Não se aplica & - & - & 6 & 9,4 & 83 & 24,3 & 7 & 26,9 & - & - & \\
\hline Não sabe/não recebeu & - & - & 5 & 7,8 & 17 & 5,0 & 4 & 15,4 & 1 & 1,8 & \\
\hline
\end{tabular}

Fonte: Almeida GM, et al., 2020. Microdados da avaliação externa do PMAQ-AB (2014). 


\section{DISCUSSÃO}

Nesta pesquisa, observou-se que no estado do Pará predominou a certificação de desempenho "Regular". Comparativamente, os estados da região sul também obtiveram a certificação "Regular", com exceção do estado de Santa Catarina, que apresentou a certificação "Bom" como mais a frequente nas ESB adscritas ao $P M A Q-A B$. Em relação à região sudeste, os estados do Espírito Santo e do Rio de Janeiro também apresentaram "Regular" como a certificação mais frequente, enquanto Minas Gerais recebeu a certificação "Bom" em maior número e o estado de São Paulo obteve o mesmo número de certificações "Bom" e "Regular" (MINISTÉRIO DA SAÚDE, 2014).

Em relação a certificação de desempenho em função do monitoramento, um estudo realizado por Mendes SR (2019) apoia os resultados encontrados nesta pesquisa. No estudo, que também utilizou dados secundários do $2^{\circ}$ ciclo do PMAQ-AB e avaliou o desempenho das ESB segundo o monitoramento da mesma, encontrou-se influência positiva na performance das ESB em todas as cinco regiões do território brasileiro que realizaram o monitoramento e análise de indicadores de saúde bucal.

Santos TP, et al. (2019) conduziram uma pesquisa semelhante na coleta descritiva dos dados do $2^{\circ}$ ciclo e encontraram que a maioria das ESB de todo território brasileiro afirmou realizar o monitoramento e análise de indicadores e informações de saúde bucal, em contradição aos resultados deste estudo quanto ao estado do Pará. Esse estudo afirmou, ainda, que o monitoramento é prática distante da rotina das equipes, porém é elemento essencial para a elaboração de critérios de reformulação do processo de trabalho baseado na identificação de situações emergenciais da população.

A prática do uso da informação para a tomada de decisões ainda é, por vezes, um desafio para gestores e profissionais no cotidiano dos serviços de saúde. A implantação de propostas que visam aprimorar a organização do processo de trabalho, envolvendo a criação de condições para a inserção da prática do monitoramento e avaliação de informações e, portanto, de indicadores de saúde como estratégia transformadora do sistema de saúde mostram-se impactantes na melhoria das condições de saúde e no desempenho dos serviços ofertados (GRIMM S, et al., 2018).

Os resultados encontrados por meio do modelo de regressão logística multinominal estão de acordo com as diretrizes propostas pela PNAB, que direciona o processo de trabalho dos atores sociais no contexto da saúde pública com a finalidade de melhorar o desempenho dos serviços ofertados. Segundo a PNAB (2017), os cirurgiões-dentistas e as ESB são responsáveis por realizar o diagnóstico epidemiológico da área de abrangência, com o objetivo de traçar o perfil do território para sistematizar e planejar a programação em saúde bucal da região. O monitoramento e a análise de indicadores de saúde bucal estão diretamente ligados a essa característica, uma vez que permitem o reconhecimento do cenário e os determinantes do processo saúde-doença, criando subsídios e norteando no processo de resolução das problemáticas existentes.

Discute-se que um efetivo planejamento em saúde bucal na atenção primária é alcançado por meio de ferramentas estratégicas que permitem visualizar a realidade e o cenário de um dado território adscrito. Destacam-se os sistemas de informação em saúde e indicadores disponíveis para os profissionais e equipes de saúde na avaliação e controle da situação de saúde, permitindo repensar e aprimorar a forma que 0 cuidado é produzido. Além disso, o acompanhamento de informações e indicadores de saúde bucal são exercícios vinculados à vigilância em saúde, prática pressuposta pela PNSB para a reorientação do modelo de atenção (FERNANDES JKB, et al., 2016; BRASIL, 2004).

Estes indicadores em saúde bucal apontam fragilidades que podem estar presentes no serviço, indicando a necessidade de atuação e resolução. Desde indicadores de primeira consulta programada, procedimentos básicos e encerramento de tratamento até indicadores de procedimentos especializados e casos suspeitos de câncer de boca. A interpretação destes indicadores é importante para que sejam alinhadas metodologias condizentes com as condições locais na elaboração de serviços e ações (ARAÚJO IDT e MACHADO FCA, 2018; FERNANDES JKB, et al., 2016).

Na perspectiva do PMAQ-AB, a prática do monitoramento de indicadores influencia na certificação de desempenho das equipes, tanto de forma direta pela própria avaliação do programa quanto pelos resultantes da qualidade de saúde bucal ofertadas aos usuários, já que provoca a criação de intervenções locais, 
regionais e nacionais que devem ser baseadas nas demandas de saúde da população, sendo portanto essencial para a implementação de medidas corretivas ou aprimoramentos das ações e serviços oferecidos pela APS (MACINKO J, et al., 2017; BRASIL, 2011).

Em relação ao apoio da gestão, infere-se que a falta da participação ativa desta no processo de incentivo para a discussão dos dados de monitoramento do sistema de informação provavelmente afeta no hábito das ESB de acompanhar e monitorar os indicadores e informações de saúde bucal, levando ao baixo percentual de equipes que o fazem segundo os achados deste estudo.

A avaliação é um instrumento de gestão. Nessa perspectiva, a institucionalização dessa prática oferece leque de opções para a tomada de decisões e a implementação de ações que atuem na realidade de saúde, que por consequência afeta positivamente no desempenho.

O monitoramento é ponto chave do processo de avaliação, cabendo à gestão local estimular esse exercício entre os profissionais de saúde. O PMAQ é, inclusive, um instrumento de grande auxílio para o incentivo de novas práticas do processo de trabalho, atuando na gestão para garantir qualidade melhora do desempenho dos indicadores de saúde (BRASIL, 2008; ARAÚJO AC, et al., 2018).

Um estudo realizado por Baldani MH et al. (2018) avaliou a desigualdade na organização do processo de trabalho das ESB por meio dos dados do PMAQ dos municípios do Paraná. Segundo o autor, foi revelado necessidade de compreensão da gestão como apoiadora das equipes do serviço de saúde bucal, dando espaço para reflexão sobre a importância e qualificação da gestão na reorganização do processo de trabalho para a correção das desigualdades intermunicipais encontradas em seus resultados.

Através dos resultados aqui encontrados, é possível visualizar o impacto do apoio da gestão sobre o desempenho que a equipe recebe na certificação, apontando a importância que esse fator possui dentro do processo de trabalho da unidade. É compromisso firmado entre o PMAQ e as gestões estaduais que exista o fortalecimento do monitoramento e avaliação com o objetivo de gerar acompanhamento e promover divulgação dos resultados da Atenção Primária à Saúde no estado, subsidiando a definição de prioridades e planejamento de ações para aprimoramento da qualidade da assistência. $O$ apoio da gestão, portanto, possui necessidade de inovação e deve dar suporte à análise de indicadores e informações de saúde (MINISTÉRIO DA SAÚDE, 2013).

Na prática, provavelmente o aproveitamento integral do uso das informações geradas pela vigilância em saúde e sua aplicação na assistência à saúde é insuficiente. Portanto, os profissionais de saúde e gestores devem estar atentos à análise dos indicadores de saúde, uma vez que estes representam grande potencial transformador das ações de saúde, impactando na qualidade do serviço oferecido ao usuário (PEREIRA BS e TOMASI E, 2016).

Os dados divulgados neste estudo revelam que o reconhecimento e o apoio do monitoramento de indicadores como uma ferramenta transformadora e fortalecedora da atenção à saúde ainda se encontra em processo de consolidação, observado por meio da carência dessa prática em grande parte das equipes de profissionais de saúde e gestores das Unidades de Atenção Primária à Saúde no estado do Pará.

Somado a isso, fica claro a influência e o impacto dessa prática no desempenho recebido pelas equipes, e, consequentemente, na saúde da população adscrita. É necessário integrar o monitoramento e análise dos indicadores como prática constante para que este possa contemplar sua finalidade de objeto norteador de intervenções no desenvolvimento das ações e atividades, servindo de instrumentos valiosos para a gestão e avaliação da situação de saúde em Atenção Primária e todos os outros níveis da Saúde Pública.

Por fim, apesar da qualidade ecológica de dados secundários deste estudo, O PMAQ-AB avaliou aproximadamente $80 \%$ de todas as ESB das cinco regiões do Brasil em seu $2^{\circ}$ ciclo, compondo banco de dados de caráter relevante para análise das equipes que trabalham na Atenção Primária à Saúde.

Portanto, o PMAQ representa um forte aliado e ferramenta de gestão para induzir mudanças na forma que o processo se dá no cotidiano dos atores sociais em saúde e na institucionalização da prática avaliativa nas atividades em saúde para incorporar a reflexão contínua da equipe sobre o seu processo de trabalho (MENDES SR, 2019; FAGUNDES DM, et al., 2018). 


\section{CONCLUSÃO}

Nota-se que a maioria das equipes estudadas no estado do Pará obteve certificação "Regular", resultado em parte pelo fato de a maioria das equipes não realizarem o monitoramento e análise dos indicadores e informações de saúde bucal e também pelo baixo apoio para discussão os dados de monitoramento dos sistemas de informação por parte da gestão. Houve diferença de certificação entre as ESB que realizaram e não realizaram monitoramento e análise dos indicadores e informações de saúde bucal. Além disso, equipes que realizaram o monitoramento apresentaram mais chance de obter melhor certificação. Este estudo contribui para a melhor compreensão da realidade do estado paraense, possibilitando a elaboração de estratégias pelos gestores, profissionais com a participação dos usuários para aprimorar o serviço de saúde atuando onde a falha está presente.

\section{REFERÊNCIAS}

1. ARAÚJO AC, et al. QUALIDADE DOS SERVIÇOS NA ESTRATÉGIA SAÚDE DA FAMÍLIA SOB INTERFERÊNCIA DO PMAQ - AB: ANÁLISE REFLEXIVA. Rev enferm UFPE. 2017; 11(2): 712-7.

2. ARAÚJO IDT, MACHADO FCA. Evolução temporal de indicadores de saúde bucal em municípios do Rio Grande do Norte. Revista Ciência Plural. 2018; 4(2): 73-86

3. BALDANI MH, et al. Processo de trabalho em saúde bucal na atenção básica: desigualdades intermunicipais evidenciadas pelo PMAQ-AB. Saúde em Debate, 2018; 42(spe1): 145-162.

4. BRASIL. Decreto ํㅜ 7.508, de 28 de junho de 2011. Regulamenta a Lei no 8.080, de 19 de setembro de 1990, para dispor sobre a organização do Sistema Único de Saúde - SUS, o planejamento da saúde, a assistência à saúde e a articulação interfederativa, e dá outras providências. Diário Oficial da República Federativa do Brasil, Brasília (DF), 2011 jun 29; Seção 1:1.

5. BRASIL. Ministério da Saúde. Diretrizes da Política Nacional de Saúde Bucal. Brasília, 2004.

6. BRASIL. Ministério da Saúde. Política Nacional de Atenção Básica. Brasília, 2017.

7. BRASIL. Secretaria de Atenção à Saúde. Análise dos Indicadores da Política Nacional de Atenção Básica no Brasil. Brasília: Ministério da Saúde, 2008.

8. E-Gestor. 2017. In: E-Gestor Atenção Básica - Informação e gestão da Atenção Básica. Ministério da Saúde. Disponível em: https://egestorab.saude.gov.br/. Acesso em: 03/04/2020

9. FAGUNDES DM, et al. Diálogos sobre o processo de trabalho em saúde bucal no Brasil: uma análise com base no PMAQ-AB. Cad. Saúde Pública. 2018; 34(9): e00049817.

10. FERNANDES JKB, et al. Avaliação dos indicadores de saúde bucal no Brasil: tendência evolutiva pró-equidade? Cad saúde pública. 2016; 32(2): 1-18.

11. FLÔRES GMS, et al. Gestão pública no SUS: considerações acerca do PMAQ-AB. Saúde debate, 2018; 42(116): 237 247.

12. FRANCA MASA, et al. Oral health indicators in the Interfederative Pacts of the Unified Health System: development in the 1998-2016 period. Rev. Odontol. UNESP, 2018; 47(1): 18-24.

13. GONÇALVES J, SAMPAIO J. O Acompanhamento de Indicadores de Saúde no Monitoramento e Avaliação da Atenção Básica: Uma Experiência no Distrito Sanitário de João Pessoa/PB. Revista Brasileira de Ciências da Saúde. 2015; 19(1): 55-60.

14. GRIMM S, et al. Dezesseis anos de monitoramento em saúde na atenção primária em uma grande metrópole das Américas. Rev Panam Salud Publica, 2018; 42(e283).

15. LIMA CA, et al. Avaliação do processo de trabalho entre equipes de saúde da família de um município de minas gerais, Brasil. Trab. Educ. Saúde. 2019 17(1): e0018710.

16. MACINKO J, et al. Brazil's National Program for Improving Primary Care Access and Quality (PMAQ). J Ambulatory Care Manage. 2017; 40(Supp 2): 4-11.

17. MENDES SR. Perfil dos cirurgiões-dentistas e sua influência sobre o desempenho das equipes de saúde bucal atuantes no sistema único de saúde brasileiro. Tese (Doutorado em Odontologia na Saúde Coletiva) - Faculdade de Odontologia. Universidade Federal de Minas Gerais, Belo Horizonte, 2019; $130 \mathrm{p}$.

18. MINISTÉRIO DA SAÚDE. Programa de Melhoria do Acesso e da Qualidade: Manual Instrutivo Para as Equipes de Atenção Básica (Saúde da Família, Saúde Bucal e Equipes parametrizadas) e NASF - 2º Ciclo. Brasília, 2013.

19. MINISTÉRIO DA SAÚDE. Programa de Melhoria do Acesso e da Qualidade: Lista de certificação por equipe - $2^{\circ}$ Ciclo. Brasília, 2014.

20. NEVES M, et al. Atenção primária à saúde bucal no Brasil: processo de trabalho das equipes de saúde bucal. Ciênc. Saúde Colet, 2019; 24(5): 1809-1820.

21. PEREIRA BS, TOMASI E. Instrumento de apoio à gestão regional de saúde para monitoramento de indicadores de saúde. Epidemiol. Serv. Saúde, 2016; 25(2): 411-418.

22. SANTOS TP, et al. What we know about management and organisation of primary dental care in Brazil. PLOS ONE, 2019; 14(4): e0215429.

23. SEIDL H, et al. Gestão do trabalho na Atenção Básica em Saúde: uma análise a partir da perspectiva das equipes participantes do PMAQ-AB. Saúde em Debate, 2014; 38: 94-108.

24. ZERMIANI TC, et al. Indicadores de desenvolvimento humano e de saúde bucal na atenção básica nos municípios da região metropolitana de Curitiba-PR. RFO UPF, 2014; 19(2): 185-192. 